\title{
Experimental and numerical investigation of thermal bridging effects of jointed Vacuum Insulation Panels
}

Alice Lorenzati, Stefano Fantucci, Alfonso Capozzoli*, Marco Perino

TEBE Research Group, Department of Energy, Politecnico di Torino, Corso Duca degli Abruzzi 24, 10129 Torino, Italy

${ }^{*}$ Corresponding author: Alfonso Capozzoli

Tel.: +39 011 0904413; fax: +390110904499

E-mail addresses: alice.lorenzati@gmail.com (A. Lorenzati), stefano.fantucci@polito.it (S. Fantucci), alfonso.capozzoli@polito.it (A. Capozzoli), marco.perino@polito.it (M. Perino)

\begin{abstract}
Vacuum Insulation Panels (VIPs) are characterised by very low thermal conductivity, compared to traditional insulating materials. For this reason, they represent a promising solution to improve the thermal behaviour of buildings, especially in the case of energy retrofitting (where a higher performance and less thickness is desirable). VIPs are insulating components in which a core material is surrounded by an air tight envelope which allows a high degree of internal vacuum to be maintained. Such features, on the one hand, allow excellent thermal insulation properties to be achieved, but, on the other, require the manufacturing of prefabricated panels of fixed shape/size. This means that the use of these super insulating materials in the building envelope involves the problem of joining the panels to each other and of fixing them onto additional supporting elements.
\end{abstract}

As a result, purposely studied supporting structures or systems are required. However, these structures and systems cause thermal bridging effects. The overall energy performance of the resulting insulation package can therefore be affected to a great extent by these additional elements, and can become significantly lower than that of the VIP panel alone.

In order to verify the incidence of thermal bridges on the overall energy performance of an insulation system that makes use of VIP panels, an experimental campaign has been carried out using a heat flux meter apparatus and analysing different joint materials/typologies. First, a measurement method was proposed, tested and verified on the basis of data from the available literature. A series of measurements on different samples was then performed. The experimental results were then used to calibrate and verify a numerical 
model that allows the performance of various "VIP packages" to be predicted and the performance of the overall package to be optimised.

Keywords: Vacuum Insulation Panel; VIP; guarded heat flux meter; numerical simulation; thermal bridges; equivalent thermal conductivity; linear thermal transmittance.

\section{INTRODUCTION}

Vacuum Insulation Panels (VIPs) represent a promising solution to reduce heat losses through a building envelope and to improve building energy efficiency. The thermal conductivity of these panels is in fact 5 to 10 times lower than that of traditional insulating materials. However, their widespread application is still hindered by their high costs, the uncertainty about their durability and the lack of experience on how to assemble the panels. Moreover, the actual performance of the system in real building applications, considering the effects of the joints between VIPs themselves and the fixing devices (laths and battens), has not yet been fully established (in this paper the term "assembly" will be used to identify the set of: VIP panels, fixing structure and joints).

In the last few years, a great number of researches have been conducted in relation to VIP technologies. Several studies have been related to the optimization of the panel properties. Some of them have focused on the analyses and development of the core material [1,2] and of the envelope configuration $[3,4,5]$. Vacuum degradation due to gas and water vapour permeation, the risk of damage (VIP perforation) and the impact of environmental conditions have also been studied frequently $[1,6,7]$. Panels with different core materials and envelopes consisting of a multilayer barrier with metalized surfaces allow thermal conductivities of about $\lambda_{c o p}=0.003-0.005 \mathrm{~W} / \mathrm{mK}$ to be achieved in the centre of the panel (depending on the core material and void degree) [2].

Examples of VIP applications for the energy refurbishment of buildings and for new constructions have been reported in [8,9]. Some studies have focused on the real performances of VIPs [3], while others have concentrated on VIP assemblies [5,10,11] and thermal bridging effects [12].

The issue of thermal bridges in technologies that make use of VIPs is very important. Thermal bridging effects can be generated from three different sources:

a) the VIP envelope alone (two panels laid in perfect, ideal contact with each other) [13];

b) the air gap between two adjacent envelopes (two mounted VIP panels lying one beside the other) [12]; 
c) the presence of structural joints, made of various materials (wood, polystyrene, aerogel...), used to couple two adjoining VIP panels (this configuration can be considered as a thermal bridge at the scale of the building envelope component) [12,14].

Thermal bridges due to the VIP envelope - case "a" - have been analytically and numerically investigated in [15], considering the four parameters that mainly influence their linear thermal transmittance, that is: laminate thickness, laminate thermal conductivity, core material thermal conductivity and panel thickness. Other researchers have proposed various solutions to reduce the thermal bridging effects for cases "b" and "c" $[16,17,18]$.

Case "c" is the typology which has the most influence on the overall thermal properties of a VIP assembly, especially as far as the vertical building envelope components are concerned. For such configurations, the installation of VIP panels necessarily requires a mounting and support system. This can be achieved (analysing the solutions available in literature) with laths and battens of different materials (such as MDF and XPS) [9], with metal and plastic rail systems [9,19], or with plaster and adhesives [9]. A new fastening method has been analysed in [20]. With this solution, VIPs have to be prefabricated with two holes (insulated or not) where dowels are later inserted to anchor the panels to the building structure. Instead, for horizontal building surfaces, VIP panels are usually laid down without any additional structural support. In this case, only air joints are of interest (case "b") [3]. In general, for mechanical protection, VIP panels need to be surrounded by two (additional) protective layers, which can also act as finishing surfaces. Such layers can play a role in enhancing or lowering thermal bridges, as highlighted in $[13,21,22]$. The effect of thermal bridging on VIP panels in real building applications has been one of the main topics of recent research activities. Sprengard and Holm [23] have investigated the effects on the overall assembly performances of: additional cover layers, VIP panel sizes and air gaps between panels (with different widths and filler materials), by means of numerical simulations. However, these studies have not covered all the possible joint configurations (e.g. thinner air gaps and structural joints between panels) and, besides, have been based on purely numerical methods.

In this frame, a research has been conducted with the aim of obtaining more detailed knowledge on the overall thermal behaviour of VIP assemblies (through the concept of "equivalent thermal conductivity", $\lambda_{e q}$ ) and of assessing the linear thermal transmittance, $\psi$, of thermal bridges. In order to achieve such goals, a method for measuring the $\psi$ parameter has been conceived, tested and verified through a comparison with literature data. After the validation, the measurement procedure was applied to experimentally analyse the dependence of the thermal bridge effects on: 
- the geometry of the air joint between two adjacent panels (e.g. size of the air gap), case "b";

- the type of structural joints (e.g. adoption of different materials), case "c";

- the panel size/shape (for both air and structural joints).

Finally, the experimental results were used to verify a numerical model. This model, once it had been verified, was used to carry out sensitivity analyses in which the geometry of the joints and their configurations were varied.

\section{ANALYSIS METHODS}

In the first phase of the research, an experimental method was proposed and tested to quantify the linear thermal transmittance of the thermal bridge between the VIP panels. This procedure was then applied to analyse the equivalent thermal conductivity of typical VIP assemblies, considering the influence of joints (either structural or air joints). Various materials, whose thermal properties influence the effects of thermal bridges, as well as several geometrical configurations, were tested. This sensitivity analysis included variations of the VIP size and shape, the thermal conductivity of the joint materials and the coupling methods (air joints or structural joints).

In a second stage of the activity, the experimental results were used to verify a numerical model that was then applied to perform sensitivity analysis by changing the joint configurations.

\subsection{Parameters used to quantify thermal bridging effects}

In order to quantify the effects of thermal bridging, two parameters were adopted: the linear thermal transmittance of the thermal bridge $-\psi$, and the equivalent thermal conductivity of the assembly $-\lambda_{e q}$. The first quantity is of general application and is useful for the design calculation. The second quantity depends not only on the thermal bridge structure and materials, but also on the shape and size of the panel; therefore, its generalization is limited. Nevertheless, $\lambda_{e q}$ is a parameter worth of note since it allows a direct and straight comparison to be made between different materials and assemblies.

\subsubsection{The linear thermal transmittance of the thermal bridge, $\psi$}

In traditional building constructions, linear thermal bridges (EN ISO 14683:2007 [24]) are likely to occur at junctions between external elements (corners of walls, walls to roofs, wall to floor), at junctions between internal walls and external walls and roofs, at junctions between intermediate floors and external walls, at columns in external walls and around windows and doors. They represent a singularity on a continuous 
homogenous surface. A similar situation occurs when VIP boards are adopted for high energy performing building envelopes. Linear thermal bridges arise at the edges of the panels, due to the necessity of coupling together panels of predetermined size and shape, and of fixing them onto the building structure (as described in Section 1). In this case, however, the overall configuration is quite different from that of traditional envelopes, where the number of linear thermal bridges is limited in a good building design. When Super Insulating Materials (SIMs) are used, in fact, a "cobweb" of distributed, but less marked, linear thermal bridges occurs. Despite this significant difference (few severe thermal bridges versus many, but milder, distributed thermal bridges), the overall effect of thermal bridges in VIP assemblies is relevant and can be evaluated by means of the so-called linear thermal transmittance of the thermal bridge, $\psi$ [26]. This parameter, according to EN ISO 10211-1:2007 [25], is defined as:

$\psi_{y}=L^{2 D(x, z)}-U_{x, y} \cdot l_{x}-U_{y, z} \cdot l_{z}$

where the symbols in Eq. (1) refer to the calculation scheme shown in Fig. 1.

$L^{2 D(x, z)}$ being the so-called "2D coupling coefficient":

$L^{2 D(x, z)}=\frac{1}{l_{y}} \frac{\dot{Q}_{a}}{\Delta \vartheta}$

$\dot{Q}_{a}$ is the overall heat flux that actually crosses the considered portion of the envelope component (with a length $I_{x}+I_{z}$ and a depth $I_{y}$ ) and it accounts for both:

- the heat flux though the zones where the panels show undisturbed behaviour,

- the heat flux through the thermal bridge and those areas of the panels in which the temperature field is influenced by the thermal bridge itself.

Owing to the 3D distortion of the temperature field, induced by the joints between the panels, the actual heat flux, $\dot{Q}_{a}$, will be higher than the one that would cross the same component if it were made of homogeneous VIP panels alone, $\dot{Q}_{1 D}$. The latter quantity could be evaluated through the well known equation:

$\dot{Q}_{1 D}=\left(U_{x, y} \cdot A_{x, y}+U_{y, z} \cdot A_{y, z}\right) \cdot\left(\vartheta_{o}-\vartheta_{i}\right)$

The difference $\Delta \dot{Q}$, between $\dot{Q}_{a}$ and $\dot{Q}_{1 D}$, is the so-called "extra-flux", that is, the additional heat transmission caused by the linear thermal bridge.

Hence, Eq. (1) can be rewritten as:

$\psi_{y}=\frac{1}{l_{y}}\left(\frac{\dot{Q}_{a}}{\Delta \vartheta}-U_{x, y} \cdot A_{x, y}-U_{y, z} \cdot A_{y, z}\right)$

It is worth noting that the value of the linear thermal bridge calculated by means of Eq. (1) or (4) depends on the geometrical configuration adopted for its estimation. Consequently, designers and engineers must 
carefully take into account how this quantity was assessed, in order to apply it correctly when developing the calculations.

In the present paper, the linear thermal transmittance has always been determined by assuming the geometrical configuration shown in Fig. 1 (the typical one, as suggested in Appendix C of the EN ISO 102111:2007 Standard [25]). However, it is also possible to choose a different configuration. For example, another geometrical configuration that is sometimes used to assess the $\psi$ parameter (see e.g. [3] and [23]) considers the symmetry plane sketched in Fig. 1 and, consequently, focuses on just one half of the building element (i.e. only the portion of area $A_{x, y}$ shown in Fig. 1 is considered). In this case, the resulting value of $\psi$ (let us call it $\psi^{\prime}$ ) will be one half of that provided by Eq. (4). In fact, assuming a symmetrical configuration (that is, $A_{x, y}=A_{y, z}=A$ and $U_{x, y}=U_{y, z}=U$ ) and applying Eq. (4) to the scheme shown in Fig. 1, it results that:

$\psi_{y}=\frac{1}{l_{y}}\left(\frac{\dot{Q}_{a}}{\Delta \vartheta}-2 \cdot U \cdot A\right)$

Instead, if only the right hand side of the envelope component is considered (e.g. only the part of area $A_{x, y}$ ), one obtains:

$\psi_{y}^{\prime}=\frac{1}{l_{y}}\left(\frac{\dot{Q}_{a} / 2}{\Delta \vartheta}-U \cdot A\right)$

Thus, the following holds:

$\psi_{y}^{\prime}=\frac{\psi_{y}}{2}$

In this paper, the linear thermal transmittance, $\psi_{y}$, has been determined by means of Eq. (5), both as far as the numerical simulations and the experimental tests are concerned.

In other words, the methodology adopted to assess $\psi$, was as follows:

- A suitable cross length of the sample (e.g. $I_{x}$ and $I_{z}$ values) was chosen in order to include the entire region in which the heat flux departs from the one-dimensional condition (e.g. undisturbed flux), according to the EN ISO 14683:2007 guidelines [24]. In particular, an area $A_{m}=254 \times 254 \mathrm{~mm}$ was assumed (which corresponds to the measurement area of the adopted experimental apparatus).

- The actual heat flux, $\dot{Q}_{a}$, was measured and/or determined by means of numerical simulations (as discussed in the following sections),

- The heat flux $\dot{Q}_{1 D}$ was determined starting from the knowledge of the centre of panel (cop) thermal conductivity $\left(\lambda_{c o p}\right)$ of the VIP boards. In fact, at a steady state, we obtain :

$$
\left|\dot{Q}_{1 D}\right|=U \cdot A \cdot\left(\vartheta_{o}-\vartheta_{i}\right)=\frac{\lambda_{c o p}}{s} \cdot A \cdot\left(\vartheta_{\text {sur }, o}-\vartheta_{\text {sur }, i}\right)
$$

- The VIP surface temperatures, $\vartheta_{\text {sur }, o}$ and $\vartheta_{\text {sur }, i}$ were used to assess the $\psi$ value. 
Therefore, the final equation adopted for the calculation was:

$\psi_{y}=\frac{1}{l_{y}}\left(\frac{\dot{Q}_{a}}{\Delta \vartheta_{s u r}}-2 \cdot \frac{\lambda_{\text {cop }}}{s} \cdot A\right)$

It is worth noting that the temperature difference between the outdoor and indoor facing surfaces, $\Delta \vartheta_{\text {sur, }}$ was used to assess $\psi$, instead of the usual temperature difference between the outdoor and the indoor environment. The reason for this choice is related to the main aim of the paper, that is, to set up an experimental procedure so as to investigate the influence of the thermal bridging effect in VIP panels and to verify numerical models (and not to assess the $\psi_{y}$ values for practical applications). Since the experimental apparatus adopted to develop the measurements (as it will be shown in the next section) was a Guarded Heat Flux Meter (GHFM) device, all the tests were conducted by imposing fixed surface temperatures on both sides of the sample under analysis, according to [27].

Consequently, $\Delta \vartheta_{\text {sur }}$ was assumed as the temperature difference for both the experimental and numerical analyses (in order to be able to directly compare theoretical and experimental results).

\subsubsection{The equivalent thermal conductivity $-\lambda_{e q}$}

The linear thermal transmittance of the thermal bridge, $\psi$, provides a useful parameter for the detailed calculation of heat losses in buildings. Nevertheless, it does not allow a straightforward comparison to be made between an ideal building envelope component, made solely of homogeneous VIP panels, and the actual components made of VIP panels and fixing devices (i.e. with the presence of thermal bridges). Such information can easily be obtained by introducing the so-called "equivalent thermal conductivity" of the assembly, $\lambda_{e q}$. This quantity is defined as:

$\lambda_{e q}=\frac{\dot{Q}_{a} \cdot s}{A \cdot \Delta \vartheta_{s u r}}$

Analogously to the assessment of the linear thermal transmittance of the thermal bridges:

- no additional layers or internal and external surface resistances were considered for the calculation of $\lambda_{\text {eq }}$

- the surface temperatures were assumed as a reference $\left(\Delta \vartheta_{\text {sur }}\right)$, instead of environmental temperatures (for the previously discussed reasons - Section 2.1.1).

It is worth noting that the equivalent thermal conductivity concept cannot be generalised and universally applied as the $\psi$ parameter. In fact, given the same materials of the VIP boards and of the joints, the value of $\lambda_{e q}$ depends on the geometrical configuration of the assembly and on the shape of the VIP panels (number and length of the thermal bridges). The main purpose of $\lambda_{e q}$ was to evaluate the influence of the VIP size/shape on the overall effect of the thermal bridges. 


\subsection{The experimental procedure}

The measurements were carried out with "LASERCOMP FOX600", a Guarded Heat Flux-Meter apparatus GHFM (Fig. 2a) that complies with the ASTMC518-91 Standard [28]. The heated/cooled plates were $600 \times 600 \mathrm{~mm}$; the measurement area was located in the centre of the plates and had a surface of $A_{m}=254 \mathrm{x}$ $254 \mathrm{~mm}$ (Fig. 2b).

The apparatus was calibrated with a "1450b NIST SRM" reference sample (certified by NIST) and an EPS reference sample (high accuracy expanded polystyrene), and was periodically verified through comparison with calibration materials. The technical specifications of the GHFM apparatus are reported in [29]. The nominal accuracy declared by the manufacturer for the measurement of the equivalent thermal conductivity is about $\pm 1 \%$. However, the actual accuracy was evaluated for each single test according to EN ISO 12667:2002 (annex B) [30], taking into consideration the actual boundary and measurement conditions. All the measurements were performed following the specification given in the EN ISO 12667:2002 international standard [30].

Heat flux meter apparatuses are usually adopted to assess the equivalent thermal conductivity of homogeneous materials. For this purpose, a board with parallel and smooth surfaces is located between two plates, which are kept at constant and different temperatures. When stable conditions are reached, the specific heat flux (average value over the centre measurement area, $A_{m}$ ) is measured.

The thermal resistance of the panel is then determined as:

$R=\frac{\Delta \vartheta_{\text {sur }}}{\varphi}$

Finally, by measuring the board thickness $(s)$ (this is done in the used apparatus by averaging the measurements at each corner of the panel), the equivalent thermal conductivity $\lambda_{c o p}$ of the material is calculated as:

$\lambda_{\text {cop }}=\frac{s}{R}$

A similar procedure was adopted to measure the actual overall heat flux, $\dot{Q}_{a}$, through two adjoining VIP panels with a thermal bridge in-between. A sample, made of two identical panels coupled by a linear thermal bridge, was introduced inside the apparatus in such a way that the measurement area was centred on the thermal bridge itself (see Fig. 2b). 
In a preliminary phase, a series of measurements was conducted to assess the $\lambda_{c o p, e x p}$ of different VIP samples, with thicknesses of 10,20 and $30 \mathrm{~mm}$, respectively (the $\lambda_{c o p, e x p}$, as seen in the previous section, is necessary for the quantification of the thermal bridge effects).

A sensitivity analysis was then performed by testing various thermal bridge configurations.

The experiments were done for each configuration and for each panel thickness (e.g., 10, 20 and $30 \mathrm{~mm}$ ), for a total of 21 tests.

All the experiments were conducted adopting two identical 600x300 mm VIP boards (symmetrical configuration) connected by means of various joint types. Specifically, the following joint materials were considered:

- Air (different air gap widths );

- Para (natural rubber);

- $\operatorname{MDF}$ (Medium Density Fibreboard);

- XPS (extruded polystyrene);

- $\quad \operatorname{ABP}($ Aerogel Based Product - blanket)

The thermal transmittances of the structural joint materials (Para, MDF, XPS and ABP) had previously been determined through the same experimental method adopted for the measurement of the VIP centre of the panel thermal conductivity. It is worth noting that Para is a material that is not normally used in VIP building applications. However, it has been included in this study in order to have the possibility of analysing cases in which the joint material is characterised by high thermal conductivity (the thermal conductivity of Para, $\lambda_{\text {Para }}$, is roughly twelve times higher than that of $A B P$ and about two times higher than that of MDF)

The width of the structural joints was kept constant for all these tests and was chosen equal to $36 \mathrm{~mm}$ (a typical value according to [31] and [12]). Instead, four different sizes of the spacers between the VIP panels were considered for the air joints. In this case, in fact, the width of the air gap has a significant influence on the thermal bridge effects.

Since the shape of the VIP panels is not regular (the edges are folded and the welded envelope is bent along the board perimeter), the effective width of the air gap is not easy to assess. In order to solve this problem, an experimental procedure, based on a photographic technique, was applied (this method is able to provide the average value of the air gap width). The experimental set up used for the measurement is shown in Fig. 3a. The panel joints were photographed using a high resolution digital camera (12.3 megapixel sensor), zooming into the control area to maximize the picture quality (Fig. 3b). The photos were 
"straightened" using a photo editing software and were then imported into a CAD software programme, to scale the images and measure the average air gap width.

The welded margins of the panels have a complex geometry (see, for example, the scheme of Fig. 3c), and the air cavity is therefore larger on one side than on the other. It was decided, for the sake of convenience, to evaluate the width of the air gap on the side in which this value is greater (this provides a safety factor, leading to slightly overestimate the influence of the thermal bridge).

The measurements were always conducted assuming a set point temperature of $35^{\circ} \mathrm{C}$ for the hot (lower) plate and of $15^{\circ} \mathrm{C}$ for the cold (upper) plate (average testing temperature: $25^{\circ} \mathrm{C}$ ) [27]. All the joints were sealed with adhesive tape, in order to avoid air exchange between the air joint and the surrounding environment.

The results of the experimental campaign are summarised in Section 3 and discussed in Section 4.

\subsection{The numerical model}

Numerical simulations were performed for the same shape/configuration as that of the tested samples, for verification purposes. A 2D numerical model was created with the Physibel BISCO software. A preliminary grid dependency analysis was performed in order to provide grid independent results (the heat flux divergence of the whole numerical model was lower than $0.1 \%$, according to the guidelines given in EN ISO 10211:2007 [25]).

The thermal conductivity values of the VIP panels - $\lambda_{c o p}$, and of the joint materials assumed for the calculations, were equal to the ones obtained from the experimental measurements.

The actual multilayer structure of the VIP envelope was simplified into a single "equivalent" homogeneous layer (this approach was already used in [3]). The thermal conductivity of this equivalent homogeneous layer was evaluated in a different way, depending on whether it was crossed by a heat flux perpendicular $(\lambda \perp)$ or parallel $\left(\lambda_{/}\right)$to the surface.

The obtained values are summarised in Fig. 4 (the thickness and the thermal conductivity of the various envelope layers were the nominal ones declared by the manufacturers).

The equivalent thermal conductivity of:

- the air gaps between two panels, in the case of structural joints (named "Still air Joints" in Fig. 5a)

- the still air between the folds of the VIP envelope (named "Still air VIP" in Fig. 5a and Fig. 5b)

used in the model were evaluated according to the EN ISO 6946:2008 guidelines (simplified calculation method) [32]. These values are summarised in Table 1. 
A total of 21 simulations were carried out, considering the same geometry and boundary conditions as those of the experimental tests.

Finally, the linear thermal transmittance of the thermal bridge and the equivalent thermal conductivity of the assemblies were determined, on the basis of the results of the numerical simulations, by applying Eq.s (9) and (10), respectively.

Table 1. Thermal conductivities of the air gaps, obtained according to the EN ISO 6946:2008 guidelines [32].

\begin{tabular}{|c|c|c|c|c|}
\hline $\begin{array}{l}\mathbf{s} \\
{[\mathrm{mm}]}\end{array}$ & $\begin{array}{l}\mathbf{d} \\
{[\mathrm{mm}]}\end{array}$ & $\begin{array}{l}\lambda_{\text {Air Joint }} \\
{[\mathrm{W} / \mathrm{mK}]}\end{array}$ & $\begin{array}{l}\lambda_{\text {Still air VIP }} \\
{[\mathrm{W} / \mathrm{mK}]}\end{array}$ & $\begin{array}{l}\lambda_{\text {Still air Joint }} \\
{[\mathrm{W} / \mathrm{mK}]}\end{array}$ \\
\hline \multirow{4}{*}{10} & 2.9 & 0.0549 & \multirow{4}{*}{0.0391} & \multirow{4}{*}{0.0391} \\
\hline & 4.5 & 0.0611 & & \\
\hline & 5.6 & 0.0631 & & \\
\hline & 8.7 & 0.0679 & & \\
\hline \multirow{4}{*}{20} & 2.7 & 0.0932 & \multirow{4}{*}{0.0612} & \multirow{4}{*}{0.0612} \\
\hline & 3.5 & 0.1110 & & \\
\hline & 5.6 & 0.1201 & & \\
\hline & 6.7 & 0.1229 & & \\
\hline \multirow{4}{*}{30} & 4.3 & 0.1682 & \multirow{4}{*}{0.0910} & \multirow{4}{*}{0.0910} \\
\hline & 5.3 & 0.1731 & & \\
\hline & 6.8 & 0.1779 & & \\
\hline & 9.3 & 0.1848 & & \\
\hline
\end{tabular}

\section{RESULTS}

\subsection{Preliminary measurements - centre of panel thermal conductivities, $\lambda_{c o p}$}

As previously mentioned, a preliminary experimental campaign was carried out to determine the thermal conductivity of the materials that constitute each single component of the assembly (VIPs + structural joint materials).

It is worth noting that the tests and/or simulations of the assembly were done considering two panels joined with each other side by side. Therefore, for each configuration two different panels were involved, each one having its own $\lambda_{c o p}$ value. The calculations were developed using a single $\lambda_{c o p}$ value, obtained by averaging the two thermal conductivities measured on the couple of VIP panels that constitutes the assemblies.

Table 2 summarises the results of these tests.

Table 2. Thermal conductivities $[\mathrm{W} / \mathrm{mK}]$ of the VIP panels ( $\lambda_{c o p}$, average value between the two panels that constitutes the assembly) and of the materials used for the structural joints.

\begin{tabular}{|c|c|c|c|c|c|c|}
\hline VIP & & & ral Joint $n$ & terial & & \\
\hline VIP $10 \mathrm{~mm}$ & VIP $20 \mathrm{~mm}$ & VIP $30 \mathrm{~mm}$ & Para & MDF & XPS & ABP \\
\hline $0.0054 \pm 0.0001$ & $0.0046 \pm 0.0001$ & $0.0048 \pm 0.0001$ & $0.2053 \pm 0.0042$ & $0.1034 \pm 0.0021$ & $0.0350 \pm 0.0007$ & $0.0166 \pm 0.0003$ \\
\hline
\end{tabular}




\subsection{Air joints - linear thermal transmittance $-\boldsymbol{\Psi}_{\text {Air Joint }}$}

The joint width $(d)$ and the panel thickness $(s)$ are the two main factors of influence on which the thermal behaviour of the air joint VIP assemblies depends. Therefore, in order to have a comprehensive picture, the analysis was carried out for four different joint widths $(d)$ and three panel thicknesses.

The four air joints analysed were named: "Best Case" (corresponding to the thinnest joint that was possible to realise in laboratory conditions), "Realistic Case" (which could represent the commonly adopted one in real building applications), "Below average Case" (for wider joints, corresponding to a poor quality of the installation), and "Worst Case" (which could be considered as a case of a faulty installation). Fig. 6 shows both the measurement and numerical results of this analysis, which allow the reliability of the simulations to be evaluated and the effect of the joint width $(d)$ on the thermal bridge to be highlighted.

Table 3. Air joints - Linear thermal transmittance $\psi$ of the thermal bridge. Experimental and numerical results for 10, 20, $30 \mathrm{~mm}$ panel thicknesses ${ }^{\prime}$.

\begin{tabular}{lllll}
\hline $\mathbf{S}$ & $\begin{array}{l}\text { Air joint } \\
\text { analysed } \\
\text { configuration }\end{array}$ & $\begin{array}{l}\mathbf{d} \\
{[\mathrm{mm}]}\end{array}$ & $\begin{array}{l}\boldsymbol{\Psi}_{\text {EXP }} \\
{[\mathrm{W} / \mathrm{mK}]}\end{array}$ & $\begin{array}{l}\boldsymbol{\Psi}_{\text {NUM }} \\
{[\mathrm{W} / \mathrm{mK}]}\end{array}$ \\
\hline \multirow{4}{*}{$\mathbf{1 0}$} & Best Case & 2.9 & $\mathbf{0 . 0 2 8 2}$ & $\mathbf{0 . 0 3 6 2}$ \\
& Realistic Case & 4.5 & $\mathbf{0 . 0 3 1 9}$ & $\mathbf{0 . 0 4 6 8}$ \\
& Below average & 5.6 & $\mathbf{0 . 0 3 8 2}$ & $\mathbf{0 . 0 5 3 7}$ \\
& Worst Case & 8.7 & $\mathbf{0 . 0 5 5 1}$ & $\mathbf{0 . 0 7 4 3}$ \\
\hline \multirow{4}{*}{$\mathbf{2 0}$} & Best Case & 2.7 & $\mathbf{0 . 0 2 5 5}$ & $\mathbf{0 . 0 2 3 6}$ \\
& Realistic Case & 3.5 & $\mathbf{0 . 0 2 8 4}$ & $\mathbf{0 . 0 2 8 4}$ \\
& Below average & 5.6 & $\mathbf{0 . 0 3 9 1}$ & $\mathbf{0 . 0 4 2 0}$ \\
& Worst Case & 6.7 & $\mathbf{0 . 0 5 1 9}$ & $\mathbf{0 . 0 4 9 0}$ \\
\hline \multirow{3}{*}{$\mathbf{3 0}$} & Best Case & 4.3 & $\mathbf{0 . 0 1 6 8}$ & $\mathbf{0 . 0 2 9 0}$ \\
& Realistic Case & 5.3 & $\mathbf{0 . 0 1 9 1}$ & $\mathbf{0 . 0 3 4 8}$ \\
& Below average & 6.8 & $\mathbf{0 . 0 2 1 4}$ & $\mathbf{0 . 0 4 4 1}$ \\
& Worst Case & 9.3 & $\mathbf{0 . 0 3 2 0}$ & $\mathbf{0 . 0 5 9 9}$ \\
\hline
\end{tabular}

\subsection{Structural joints - linear thermal transmittance $-\boldsymbol{\Psi}_{\text {Joint }}$}

The thermal bridge caused by structural joints is mainly characterized by the thermal resistance of the joint, $R_{\text {Joint }}$ (e.g. by the materials that constitutes the joint itself).

Table 4 summarizes the linear thermal transmittance obtained by means of the numerical models and of the measurements as a function of the panel thickness and of the joint material (whose values determine the overall $R_{\text {Joint }}$.

\footnotetext{
${ }^{1}$ It is worth mentioning, in relation to the measured values, that it is virtually impossible to obtain different samples with exactly the same joint width, $(d)$. Owing to the rather irregular shape of the panel edges, the average width can only be made similar but not identical. This fact explains why, in Table 5, Table 6, Fig. 6 and in other comparisons, the data are gathered by ranges of the $(d)$ value (e.g. the "Realistic case" gathers data of samples with joint average widths variable from 3.5 to $5.3 \mathrm{~mm}$ ). The value of (d) that is shown in the tables and figures is the average width measured by means of the procedure outlined in Section 2.3.
} 
Table 4. Structural joints - Linear thermal transmittance $\psi$ of the thermal bridge. Experimental and numerical results for 10, 20, $30 \mathrm{~mm}$ panel thicknesses.

\begin{tabular}{|c|c|c|c|}
\hline $\begin{array}{l}\mathbf{s} \\
{[\mathrm{mm}]}\end{array}$ & $\begin{array}{l}\text { Joint } \\
\text { Material }\end{array}$ & $\begin{array}{l}\boldsymbol{\Psi}_{\mathrm{EXP}} \\
{[\mathrm{W} / \mathrm{mK}]}\end{array}$ & $\begin{array}{l}\Psi_{\mathrm{NUM}} \\
{[\mathrm{W} / \mathrm{mK}]}\end{array}$ \\
\hline \multirow{4}{*}{10} & $\mathrm{ABP}$ & 0.0753 & 0.0662 \\
\hline & XPS & 0.1244 & 0.1305 \\
\hline & MDF & 0.3109 & 0.3672 \\
\hline & Para & 0.4863 & 0.7181 \\
\hline \multirow{4}{*}{20} & $\mathrm{ABP}$ & 0.0476 & 0.0372 \\
\hline & XPS & 0.0787 & 0.0701 \\
\hline & MDF & 0.2031 & 0.1903 \\
\hline & Para & 0.3491 & 0.3705 \\
\hline \multirow{4}{*}{30} & $\mathrm{ABP}$ & 0.0331 & 0.0264 \\
\hline & XPS & 0.0529 & 0.0486 \\
\hline & MDF & 0.1403 & 0.1291 \\
\hline & Para & 0.2384 & 0.2493 \\
\hline
\end{tabular}

Fig. 7 shows the linear thermal transmittance of all the considered panels (e.g. 10, 20, $30 \mathrm{~mm}$ ) as a function of the joint thermal resistance (calculated as the ratio of the joint thickness $(s)$, to the material thermal conductivity).

\subsection{Influence of form factor on the VIPs assembly performances - equivalent thermal}

\section{conductivity $-\lambda_{\text {eq }}$}

It is a common practice to assume "practical" values of the equivalent thermal conductivity, $\lambda_{\text {eq practical, }}$ of the VIPs assembly for thermal/energy calculations in buildings. These values take into account - in a very simplified way - the overall effects of the thermal bridges regardless of the shape/size of the VIP panels and the joint type (see e.g. [12]). For example, in [34], the following suggestions are given:

- $\lambda_{\text {eq practical }}=0.007 \mathrm{~W} / \mathrm{mK}$ (for panel sizes greater than $500 \times 500 \mathrm{~mm}$ )

- $\quad \lambda_{\text {eq practical }}=0.008 \mathrm{~W} / \mathrm{mK}$ (for smaller VIPs).

This approach dramatically simplifies the design procedure, but it does not take into consideration that the equivalent thermal conductivities, $\lambda_{\text {eq }}$, of the assembly show a significant dependence on the size and shape of the VIP panels, as well as on the joint configuration. Therefore, in order to verify the reliability of such a procedure, an analysis was conducted of the influence of the "form factor" of the assembly and of the panel size. The form factor is defined as the $P / A$ ratio, where $P$ is the semi-perimeter of the panel and $A$ is the area of the panel.

Fig. 8 shows the results, obtained from the experimental and numerical analyses, of an assembly made of VIP panels with a thickness of $20 \mathrm{~mm}$ and with different form factors. 
Data for air joints - "Realistic Case", structural MDF, XPS and ABP joints are presented. The vertical dotted lines in the figure represent the size of the standard panels that are usually available on the market. The horizontal single and double dotted dashed lines highlight the $\lambda_{\text {eq practical }}$ and the measured $\lambda_{\text {cop }}$ values, respectively.

The results related to VIP panels with thicknesses of $10 \mathrm{~mm}$ and $30 \mathrm{~mm}$ are summarised in Table 5 and Tale 6.

Table 5. Influence of panel size/shape on $\lambda_{e q}$. Values derived from the experimental results. The $\left(^{*}\right)$ values highlight the VIP panel size usually adopted in building applications (data in bold are the measured ones).

\begin{tabular}{|c|c|c|c|c|c|c|c|c|}
\hline \multirow[b]{3}{*}{$\mathbf{P} / \mathbf{A}$} & \multicolumn{5}{|l|}{$10 \mathrm{~mm}$} & \multicolumn{3}{|l|}{$30 \mathrm{~mm}$} \\
\hline & \multirow{2}{*}{$\begin{array}{l}\text { AIR JOINT } \\
\mathrm{d}=4.5 \mathrm{~mm}\end{array}$} & \multicolumn{3}{|c|}{ STRUCTURAL JOINT } & \multirow{2}{*}{$\begin{array}{l}\text { AIR JOINT } \\
d=5.3 \mathrm{~mm}\end{array}$} & \multicolumn{3}{|c|}{ STRUCTURAL JOINT } \\
\hline & & $\mathrm{ABP}$ & XPS & MDF & & $\mathrm{ABP}$ & XPS & MDF \\
\hline$[1 / \mathrm{m}]$ & \multicolumn{8}{|c|}{$\lambda_{e q}[\mathrm{~W} / \mathrm{mK}]$} \\
\hline 6.67 & 0.0075 & 0.0104 & 0.0137 & 0.0261 & 0.0086 & 0.0076 & 0.0171 & 0.0346 \\
\hline 5.00 & 0.0070 & 0.0091 & 0.0116 & 0.0209 & 0.0077 & 0.0063 & 0.0134 & 0.0265 \\
\hline $3.33^{*}$ & 0.0064 & 0.0079 & 0.0095 & 0.0157 & 0.0067 & 0.0049 & 0.0097 & 0.0184 \\
\hline $2.50^{*}$ & 0.0062 & 0.0072 & 0.0085 & 0.0131 & 0.0062 & 0.0043 & 0.0079 & 0.0144 \\
\hline 1.67 & 0.0059 & 0.0066 & 0.0074 & 0.0105 & 0.0057 & 0.0036 & 0.0060 & 0.0104 \\
\hline 1.33 & 0.0058 & 0.0064 & 0.0070 & 0.0095 & 0.0056 & 0.0034 & 0.0053 & 0.0088 \\
\hline
\end{tabular}

Table 6. Influence of panel size/shape on $\lambda_{e q}$. Numerical results. The $\left(^{*}\right)$ values highlight the VIP panel size usually adopted in building applications.

\begin{tabular}{|c|c|c|c|c|c|c|c|c|}
\hline \multirow[b]{3}{*}{$\mathbf{P} / \mathbf{A}$} & \multirow{2}{*}{$\begin{array}{l}10 \mathrm{~mm} \\
\text { AIR JOINT }\end{array}$} & & & & \multicolumn{4}{|l|}{$30 \mathrm{~mm}$} \\
\hline & & \multicolumn{3}{|c|}{ STRUCTURAL JOINT } & \multirow{2}{*}{$\begin{array}{l}\text { AIR JOINT } \\
d=5.3 \mathrm{~mm}\end{array}$} & \multicolumn{3}{|c|}{ STRUCTURAL JOINT } \\
\hline & $\mathrm{d}=4.5 \mathrm{~mm}$ & $A B P$ & XPS & MDF & & $\mathrm{ABP}$ & XPS & MDF \\
\hline$[1 / \mathrm{m}]$ & \multicolumn{8}{|c|}{$\lambda_{e q}[\mathrm{~W} / \mathrm{mK}]$} \\
\hline 6.67 & 0.0085 & 0.0098 & 0.0141 & 0.0298 & 0.0118 & 0.0101 & 0.0145 & 0.0306 \\
\hline 5.00 & 0.0077 & 0.0087 & 0.0119 & 0.0237 & 0.0100 & 0.0087 & 0.0121 & 0.0242 \\
\hline $3.33^{*}$ & 0.0069 & 0.0076 & 0.0097 & 0.0176 & 0.0083 & 0.0074 & 0.0096 & 0.0177 \\
\hline $2.50^{*}$ & 0.0065 & 0.0070 & 0.0086 & 0.0145 & 0.0074 & 0.0068 & 0.0084 & 0.0145 \\
\hline 1.67 & 0.0061 & 0.0065 & 0.0075 & 0.0115 & 0.0065 & 0.0061 & 0.0072 & 0.0112 \\
\hline 1.33 & 0.0060 & 0.0062 & 0.0071 & 0.0103 & 0.0062 & 0.0058 & 0.0067 & 0.0100 \\
\hline
\end{tabular}

\section{CRITICAL DISCUSSION AND ANALYSIS OF THE RESULTS}

\subsection{Air and structural joints - linear thermal transmittance $-\psi_{\text {Air Joint }}$}

\subsubsection{Air joints}

Analysing the effect of the size of the air joint (i.e. its width) on the thermal bridge, it is possible to see (Fig. 6) that the linear thermal transmittance increases - as expected - as "d" becomes larger. 
It is worth noting that the results show an almost linear dependence of $\psi$ as a function of the joint width. The numerical data are aligned along three almost perfect parallel straight lines (darker symbols in Fig. 6), with a practically constant difference between the three panel thicknesses $\left(\Delta \psi_{\text {NUM }} 10-20 \mathrm{~mm}=0.0108 \mathrm{~W} / \mathrm{mK}\right.$, $\Delta \psi_{\text {NUM_20-30mm }}=0.0040 \mathrm{~W} / \mathrm{mK}$ and $\left.\Delta \psi_{\text {NUM_10-30mm }}=0.0148 \mathrm{~W} / \mathrm{mK}\right)$.

The experimental results ( $\psi_{\text {EXP }}$, grey symbols in Fig. 6) also show a fairly good linear behaviour of $\psi$ as a function of $(d)$, even though, in this case, the lines present slightly different slopes (the lines are somewhat less steep than those of the numerical data, especially for the 10 and $30 \mathrm{~mm}$ panels).

As far as the comparison between the experimental and numerical values of $\psi$ is concerned, it is possible to see that a non-homogenous situation arises.

A striking agreement between the predicted and measured data is found for the panel with a thickness of 20 $\mathrm{mm}$ (both in terms of absolute values and of slope of the curve $\psi(d)$ ). On the contrary, quite significant discrepancies are found for the panels with thicknesses of 10 and $30 \mathrm{~mm}$, with systematically higher predicted values than the measured ones. Moreover, for these samples, the slope of the curve also appears to be slightly different, with the measured data showing a less pronounced variation of $\psi$ as the joint size increases.

Such behaviour could be incidental, but it is worth mentioning that similar outcomes have also been found in Ghazi Wakili et al. [3], in Brunner et al. [33] and, more recently, in Spreghard and Holme [35]; this picture supports the assumption that an objective cause could be responsible for this trend.

For example, Fig. 9 shows the measured and calculated linear thermal transmittance of the thermal bridge determined on the basis of the results found in the present study (Fig. 9a) and compares it with the outcomes of [3] (Fig. 9b). Though a direct comparison between the absolute values of $\psi$ in Fig. 9a and in Fig $9 b$ is not possible, due to some differences in the testing conditions in the two researches, it is however interesting to observe how the discrepancy between the experimental and numerical results is conspicuously similar.

Some hypotheses can be made to explain why the results related to the $20 \mathrm{~mm}$ thick panel are in better agreement than those for other panel thicknesses.

First, the width of the air joint used for the calculation is a constant value that is equal to the average value (measured along the entire panel edge) of the local widths of the real sample (as sketched in Fig. 10). Two identical configurations, with the same average joint width, but significant local differences of the air gaps between the panels, do not necessarily behave in the same way when tested in the lab (although they do 
provide the same results when simulated numerically). This is primarily due to convection and radiation, which are highly non-linear phenomena.

Moreover, the measurement of the width of the air is characterized by a consistent uncertainty (due to the photographic survey method) and, again, this factor can determine a discrepancy between predicted and measured quantities.

At this stage of the research, it is impossible to clearly determine whether these discrepancies are a consequence of measurement inaccuracies (of thermal conductivities and/or of the air joint widths) or of the lack of reliability of the simplified calculation methods [32]. Further investigations are still needed to clarify such an issue.

\subsubsection{Structural joints}

Both the experimental and the numerical results are distributed along similar curves that have a power law shape (see, for example, Fig. 7). The measured and numerical values of the linear thermal transmittance are in a good agreement with each other, although the prediction accuracy is better when the thermal resistance of the joint is higher. The average percentage difference between these values is about $11 \%$, with a maximum of $50 \%$ for the Para joint in the $10 \mathrm{~mm}$ thickness panels. However, this configuration, as explained in the previous sections, has here been considered just for sensitivity analysis purposes (since such a material is not used in practical applications).

For these reasons, the numerical model can be considered reliable for cases in which $R_{\text {Joint }}>0.100 \mathrm{~m}^{2} \mathrm{~K} / \mathrm{W}$, and can consistently be applied ${ }^{2}$ to estimate the thermal bridge effect of structural joints in typical building applications.

\subsection{Influence of form factor on the VIPs assembly performances - equivalent thermal conductivity $-\lambda_{\text {eq }}$}

Fig. 8 shows that the difference between the experimental and numerical results of $\lambda_{e q}$ is rather small and tends to increase as the panel size decreases. A possible explanation of this behaviour is that the influence of the edge effect increases for smaller sizes and that the uncertainties are mainly due to edge effects.

\footnotetext{
${ }^{2}$ It should be recalled that the data shown in Table 3 and Table 4 and Fig. 6 and Fig. 7 cannot be used directly for practical applications, since they do not include the effects of the internal and external surface heat transfer coefficients.
} 
The difference between the calculated values and the values derived from the measurements for the structural joints ranges from: $4 \%(1500 \times 1500 \mathrm{~mm})$ to $6 \%(300 \times 300 \mathrm{~mm})$ for MDF, $4 \%(1500 \times 1500 \mathrm{~mm})$ to

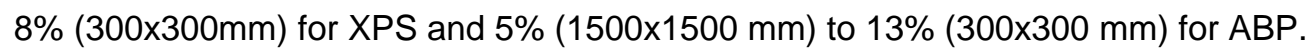

Instead, the measured and simulated data for the air joints are almost coincident. As already observed for the $\psi$ value (Section 4.1), the discrepancy between the measured and calculated values increases for panels with thicknesses of 10 and $30 \mathrm{~mm}$ (see also Table 5 and Table 6).

Obviously, there is a noticeable difference between the $\lambda_{e q}$ of the assembly and the $\lambda_{c o p}$ of the homogeneous VIP panel. This difference increases, as can be expected :

- as the thermal resistance of the joints becomes smaller (e.g. changing the materials of the joint and switching from ABP to MDF), but keeping the form factor constant,

- by decreasing the panel size (e.g increasing the form factor and, hence, the incidence of the thermal bridge on the overall assembly).

The difference between $\lambda_{e q}$ and $\lambda_{c o p}$ for typical building applications, that is, VIP sizes of $600 \times 600 \mathrm{~mm}$ and $600 \times 1200 \mathrm{~mm}$, is of about:

- $55-40 \%$ in the case of an ABP joint (respectively for the two sizes),

- $300-220 \%$ in the case of an MDF joint (respectively for the two sizes).

The lowering of the overall thermal performance of the assembly, passing from large to small sizes of the panels and from low (e.g square panels) to high (e.g long rectangular panels) P/A ratios, can be seen to be significant (Fig. 8). Therefore, in order to optimize the energy performance of the VIP technology in building applications, designers and practitioners should pay attention to the shape and the size of the panels they adopt.

The largest and squarest possible panels should be chosen, compatibly with the geometrical constraints. The combination of ABP and VIP technologies shows a remarkable potential. The low thermal conductivity of $A B P$ joints reduces the difference in performance between the air joint (the best one) and the structural joints (but this, on the other hand, implies an increase in costs) [14].

Finally, and the most important from the practical point of view, the comparison between the actual value of $\lambda_{e q}$ and the value usually recommended for thermal calculation, $\lambda_{\text {eq practical, }}$, has provided interesting conclusions. As it is possible to see, from Fig. 8 and from the data in Tables 5 and 6 , there can be very large discrepancies between $\lambda_{e q}$ and $\lambda_{e q \text { practical. }}$

The use of $\lambda_{\text {eq practical }}$ for P/A aspect ratios of VIP panels that exceed a value of about 5 leads to an overestimation of the energy performance of an assembly, as its thermal conductivity is underestimated. 
This error is not negligible, especially in the case of structural joints (particularly when a poorly performing material, such as MDF or wood, is used).

Looking at the range of form factors, $P / A$, which is the most frequently employed form factor in real building applications (e.g. the grey area in Fig. 8), the adoption of the $\lambda_{\text {eq practical }}$ value instead of a purposefully calculated value, $\lambda_{\text {eq }}$, can generate either an underestimation or an overestimation of the VIP performances, depending on the joint type. The practical values of $0.007 \mathrm{~W} / \mathrm{mK}$ and $0.008 \mathrm{~W} / \mathrm{mK}$ are overly optimistic about the assembly behaviour for all the structural joints, with the exception of the ABP joint (the actual equivalent thermal conductivity, in fact, could be even twice this value). On other hand, they tend to underestimate the behaviour of those assemblies that make use of well manufactured air joints (e.g. smaller ( $d$ ) values). However, it is important to remember that such thermal bridges in actual building applications are likely to be attenuated by the presence of additional thermal resistances due to those layers that surround the VIP panels. For this reason, the in situ values of $\lambda_{e q}$ could be lower than those obtained in the present work, in which "bare" VIP panels have been considered.

These outcomes would seem to suggest avoiding the adoption of easy-to-use, but excessively approximated, "engineering" values of the thermal properties of VIP assemblies.

In order to provide a reliable picture of the energy behaviour of buildings, the specific geometrical configuration of the VIP assembly needs to be studied carefully through numerical or experimental analyses.

\subsection{Practical considerations}

Considering the outcomes discussed in the previous sections, some practical suggestions and rules of thumb can be derived.

As illustrated in Section 4.1, a rather accurate and identifiable functional dependence of the $\psi$ value on the equivalent thermal resistance of the structural joint, $R_{\text {Joint, }}$, has been found (Fig. 7 ). Such a feature allows the performance of various materials to be compared directly on a single plot.

This suggests that, in the future, suitable, easy-to-use, but still accurate charts could be prepared and supplied to building designers to develop energy analyses.

An important issue that needs to be highlighted is that the values of the $\psi$ and $\lambda_{e q}$ parameters shown in the figures and tables presented in this paper cannot be applied directly to develop practical calculations. In fact, all the presented values refer to a theoretical case in which the internal and external surface thermal resistances have been neglected (the reason these resistances have not been included in this study are explained in Section 2). 
Finally, as clearly highlighted in Fig. 7 and Fig. 8, the performance of building envelope components that make use of VIP panels can be improved by:

- Adopting suitable materials to create the joints and fixtures of the VIP panels. Wood and wood derived materials, thought very common, cheap and widely adopted in the construction sector, should be avoided. They, in fact, can determine a significant degradation of the thermal performance of the VIP assemblies compared to homogeneous VIP panels. Aerogel materials (ABP) have proved to be a very effective solution for the optimization of the thermal performance of VIP assemblies (though they cost more).

- Adopting, whenever possible, VIP panels with the largest size and squarest shape. The $\lambda_{e q}$ of the assembly, in fact, has a rather strong linear dependence on the form factor $P / A$.

\section{CONCLUSIONS}

Vacuum Insulations Panels are a promising technology that can effectively help to reduce the energy demand of the building sector. In particular, they provide good opportunities in the field of the energy retrofitting of the existing building stock and in the realization of highly performing construction elements. Nevertheless, being a relatively new and innovative insulation material (at least as far as building applications are concerned), a number of barriers hinder their widespread application.

Cost is one of the limiting factors, even though the predictable future growth of the market suggests that a significant reduction in costs might be expected. Moreover, the savings of useful space, obtainable thanks to their decreased thickness compared to traditional insulation materials, could represent an economic benefit that could help counterbalance the higher investment costs.

Technological and cultural barriers may also have a non-negligible influence on the widespread use of VIPs. First, as demonstrated in this paper and in other studies reported in literature, the standardised procedures generally used for labelling the properties of traditional insulation materials show some weaknesses when applied to VIP panels (whose equivalent thermal conductivity is around one tenth that of other substances). The procedures, testing conditions, reliability/accuracy of the measurements should be analysed carefully and new standards should be proposed.

In addition to the labelling of the "raw" materials (e.g. the assessment of the properties of the VIP panels alone), another challenge, which is still partly unsolved, is represented by the assessment of the overall performances of thermal insulation systems that make use of VIPs (called "assemblies" in this paper). 
In other words, the evaluation of the thermal bridge effect and the standardization of the procedures adopted to perform such a calculation and/or measurement seem to be of paramount importance.

Possible mistakes could arise due to different geometrical schematizations of the VIP package. Depending on the choice of the investigator, either a $\psi_{y}$ or a $\psi_{y}{ }^{\prime}$ value may be obtained, as the relation between these two parameters is: $\psi_{y}^{\prime}=\psi_{y} / 2$. Therefore, large errors could occur if these quantities are not interpreted correctly and applied coherently by professional engineers and designers. A general agreement is highly recommended.

Numerical models have resulted to be sufficiently reliable for the analysis of structural joints (especially when the thermal resistance of the joint is larger than $0.100 \mathrm{~m}^{2} \mathrm{~K} / \mathrm{W}$ ). On the contrary, their performances may vary significantly when they are applied to the study of air joints. For the air joints, as also experienced by other investigators, excellent results have been obtained for VIP panels with a thickness of $20 \mathrm{~mm}$, while the results related to 10 and $30 \mathrm{~mm}$ thick panels have been far less accurate. Such behaviour has systematically been encountered in various researches presented in the literature. However, no satisfactory reasons have been found to explain this lack of accuracy so far. A future research activity should be devoted to investigating this trend.

Finally, but of no less importance, is the issue linked to the use of practical values for the calculation of building energy performances (e.g. $\lambda_{\text {eq, practical }}$ equal to $0.007 \mathrm{~W} / \mathrm{mK}$ or to $0.008 \mathrm{~W} / \mathrm{mK}$ ), which globally accounts for the effect of thermal bridges. It has been shown that such constant values could lead to large errors (either over or under estimations), hence it is strongly advisable not to follow this highly simplified procedure.

\section{ACKNOWLEDGEMENTS}

The research activity presented in this paper was conducted in the frame of a research contract between ENEA and the Politecnico di Torino, Department of Energy ( "Ricerca di sistema elettrico" - Par 2015 Attività B) and is part of the IEA - Annex 65 - " Long-Term Performance of Super-Insulation in Building Components \& Systems " project. 


\section{NOMENCLATURE}

$\begin{array}{lll}\lambda_{c o p} & \text { Centre of panel thermal conductivity } & {[\mathrm{W} / \mathrm{mK}]} \\ \lambda_{e q} & \text { Equivalent thermal conductivity of a VIP assembly considering } & \\ & \text { thermal bridging effects } & {[\mathrm{W} / \mathrm{mK}]}\end{array}$

$\lambda_{\text {eq practical }} \quad$ Suggested values of the equivalent thermal conductivity of a VIP assembly considering thermal bridging effects $\quad[\mathrm{W} / \mathrm{mK}]$

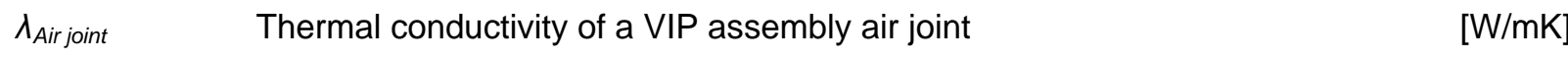

$\lambda_{\text {Still air VIP }} \quad$ Thermal conductivity of air caught between a VIP assembly air joint $\quad[\mathrm{W} / \mathrm{mK}]$

$\begin{array}{lll}\lambda_{\text {Still air joint }} \quad \text { Thermal conductivity of a VIP assembly air joint } & {[\mathrm{W} / \mathrm{mK}]}\end{array}$

$\lambda_{\perp} \quad$ Thermal conductivity of the equivalent layer $\quad$ (perpendicular heat flux) $\quad[\mathrm{W} / \mathrm{mK}]$

$\lambda_{/ /} \quad$ Thermal conductivity of the equivalent layer $\quad$ (parallel heat flux) $\quad[\mathrm{W} / \mathrm{mK}]$

$\psi \quad$ Linear thermal transmittance $\quad[\mathrm{W} / \mathrm{mK}]$

$\psi_{y} \quad$ Linear thermal transmittance along y axis $\quad[\mathrm{W} / \mathrm{mK}]$

$\begin{array}{lll}\psi_{y}^{\prime} & \text { Half of the linear thermal transmittance } & {[\mathrm{W} / \mathrm{mK}]}\end{array}$

$\begin{array}{lll}\psi_{\text {Air joint }} \quad \text { Linear thermal transmittance (air joint) } & {[\mathrm{W} / \mathrm{mK}]}\end{array}$

$\psi_{\text {Joint }} \quad$ Linear thermal transmittance (structural joint) $\quad[\mathrm{W} / \mathrm{mK}]$

$\begin{array}{lll}\psi_{\text {EXP }} & \text { Linear thermal transmittance - Measured value } & {[\mathrm{W} / \mathrm{mK}]}\end{array}$

$\psi_{\text {NUM }} \quad$ Linear thermal transmittance - Calculated value $\quad[\mathrm{W} / \mathrm{mK}]$

$\begin{array}{lll}L^{2 D(x, z)} & 2 \mathrm{D} \text { coupling coefficient } & {[\mathrm{W} / \mathrm{mK}]}\end{array}$

$U \quad$ Thermal transmittance $\quad\left[\mathrm{W} / \mathrm{m}^{2} \mathrm{~K}\right]$

I Length [m]

A Surface area $\quad\left[\mathrm{m}^{2}\right]$

$\dot{Q} \quad$ Heat flux [W]

$\varphi \quad$ Specific heat flux $\quad\left[\mathrm{W} / \mathrm{m}^{2}\right]$

$\begin{array}{lll}\vartheta_{0} & \text { Outdoor temperature } & {\left[{ }^{\circ} \mathrm{C}\right]}\end{array}$

$\vartheta_{i} \quad$ Indoor temperature $\quad\left[{ }^{\circ} \mathrm{C}\right]$

$\vartheta_{\text {sur }, 0} \quad$ Surface temperature - outdoor side $\quad\left[{ }^{\circ} \mathrm{C}\right]$

$\vartheta_{\text {sur, } i} \quad$ Surface temperature - indoor side $\quad\left[{ }^{\circ} \mathrm{C}\right]$

$R \quad$ Thermal resistance $\quad\left[\mathrm{m}^{2} \mathrm{~K} / \mathrm{W}\right]$

$P \quad$ Semi-perimeter of the/a panel [m]

$P / A \quad$ Form factor $\quad\left[\mathrm{m}^{-1}\right]$ 


\section{REFERENCES}

[1] H. Simmler, S. Brunner, Vacuum insulation panels for building application. Basic properties, aging mechanisms and service life, Energy and Buildings 37 (2005) 1122-1131. doi:10.1016/j.enbuild.2005.06.015 [2] J.-S. Kwon, C.H. Jang, H. Jung, T.-H. Song, Effective thermal conductivity of various filling materials for vacuum insulation panels, International Journal of Heat and Mass Transfer 52 (2009) 5525-5532. doi:10.1016/j.ijheatmasstransfer.2009.06.029.

[3] K. Ghazi Wakili , R. Bundi, B. Binder, Effective thermal conductivity of vacuum insulation panels, Building Research \& Information $32: 4$ (2004) 293-299. doi:10.1080/0961321042000189644.

[4] T. Nussbaumer, R. Bundi, Ch. Tanner, H. Muehlebach, Thermal Analysis of a wooden door system with integrated vacuum insulation panels, Energy and Buildings 37 (2005) 1107-1113. doi:10.1016/j.enbuild.2004.11.002.

[5] T. Nussbaumer, K. Ghazi Wakili, Ch. Tanner, Experimental and numerical investigation of the thermal performance of a protected vacuum-insulation system applied to a concrete wall, Applied Energy 83 (2006) 841-855. doi:10.1016/j.apenergy.2005.08.004.

[6] S. Brunner, K. G. Wakili, Hints for an additional aging factor regarding the thermal performance of vacuum insulation panels with pyrogenic silica core, Vacuum 100 (2014) 4-6. doi:10.1016/j.vacuum.2013.07.033.

[7] S. Brunner, K. G. Wakili, T. Stahl, B. Binder, Vacuum insulation panels for building applicationsContinuous challenges and developments, Energy and Buildings 85 (2014) 592-596. doi:10.1016/j.enbuild.2014.09.016.

[8] S. Brunner, H. Simmler, In situ performance assessment of vacuum insulation panels in a flat roof construction, Vacuum 82 (2008) 700-707. doi:10.1016/j.vacuum.2007.10.016.

[9] P. Johansson, Vacuum insulation panels in buildings - literature review, in: Report in Building Physics, 2012:1, Chalmers University of Technology, Gothenburg, 2012.

[10] R. Beatens, B.P. Jelle, J.V. Thue, M.J. Tenperik, S. Grynning, S. Uvsløkk, A. Gustavsen, Vacuum insulation panels for building application. A review and beyond, Energy and Buildings 42 (2010) 147-172. doi:10.1016/j.enbuild.2009.09.005.

[11] M.J. Tenpierik, H. Cauberg, Analytical models for calculating thermal bridge effects caused by thin high barrier envelopes around Vacuum Insulation Panels, Journal of Building Physics, January 2007 vol. 30 no. 3 185-215. 
[12] A. Lorenzati, S. Fantucci, A. Capozzoli, M. Perino, The effect of different materials joint in Vacuum Insulation Panels, SeB - 14, Cardiff, 2014. Energy Procedia 2014; 62:374-381. doi:10.1016/j.egypro.2014.12.399

[13] Capozzoli A., Fantucci S., Favoino F., Perino M., Vacuum Insulation Panels: Analysis of the Thermal Performance of Both Single Panel and Multilayer Board. Energies 2015; 8: 2528-2547. doi:10.3390/en8042528.

[14] A. Lorenzati, S. Fantucci, A. Capozzoli, M. Perino, Coupling VIPs and ABPs: assessment of overall thermal performance in building wall insulation, $6^{\text {th }}$ IBPC 2015, Torino, 2014. Energy Procedia 2015, 'In press'.

[15] M.J. Tenpierik, W. van der Spoel, J.J.M. Cauberg, Analytical models for predicting thermal bridge effects due to Vacuum Insulation Panel barrier envelopes, Bauphysik 30 (2008) 38-45.

[16] M.J. Tenpierik, H. Cauberg, Encapsulated Vacuum Insulation Panels: theoretical thermal optimization, Building Research \& Information 38(6) (2010) 660-669.

[17] T.I. Thorsell, I. Källebrink, Edge loss minimization in vacuum insulation panels, in Jóhannesson, G. (Eds.), Proceedings of the 7th Nordic Building Physics Symposium, 2005, pp. 945-952.

[18] K. Ghazi Wakili, T. Stahl, S. Brunner, Effective thermal conductivity of a staggered double layer of vacuum insulation panels, Energy and Buildings 43 (2011) 1241-1246. doi:10.1016/j.enbuild.2011.01.004. [19] H. Sallée, D. Quenard, E. Valenti, M. Galan, VIP as thermal breaker for internal insulation system, Energy and Buildings 85 (2014) 631-637. doi:10.1016/j.enbuild.2014.08.039.

[20] I. Mandilaras, I. Atsonios, G. Zannis, M. Founti, Thermal performance of a building envelope incorporating ETICS with vacuum insulation panels and EPS, Energy and Buildings 85 (2014) 654-665. doi:10.1016/j.enbuild.2014.06.053.

[21] A. Binz, A. Moosmann, G. Steinke, U. Schonhardt, F. Fregnan, H. Simmler, et al., Vacuum insulation in the building sector. Systems and application (Subtask B), Final Report for the IEA/ECBCS Annex 39 HiPTI-project High performance thermal insulation for buildings and building systems, 2005.

[22] F. Isaia, S. Fantucci, A. Capozzoli, M. Perino, Vacuum Insulation Panels: thermal bridging effects and energy performance in real building applications, SEB15, Energy Procedia (2015), 'In press'.

[23] C. Sprengard, A.H. Holm, Numerical examination of thermal bridging effects at the edges of vacuuminsulation-panels (VIP) in various constructions, Energy and Buildings 85 (2014) 638-643. doi:10.1016/j.enbuild.2014.03.027 
[24] EN ISO 14683:2007, Thermal bridges in building construction - Linear thermal transmittance Simplified methods and default values, European Committee for Standardization, 2007.

[25] EN ISO 10211:2007. Thermal bridges in building construction - Heat flows and surface temperatures - Detailed, European Committee for Standardization, 2007.

[26] A. Capozzoli, A. Gorrino, V. Corrado, A building thermal bridges sensitivity analysis, Applied Energy 107 (2013) 229-243. doi:10.1016/j.apenergy.2013.02.045.

[27] A. Lorenzati, S. Fantucci, A. Capozzoli, M. Perino, VIPs thermal conductivity measurement: test methods, limits and uncertainty, $6^{\text {th }}$ IBPC 2015, Energy Procedia (2015), 'In press'.

[28] ASTM International. ASTM C518-91 Test Method for Steady-State Heat Flux Measurements and Thermal Transmission Properties by Means of the Heat Flow Meter Apparatus, 1991.

[29] LaserComp, Inc., @, FOX600 and FOX800 Series Instruments Manual, 2001-2005. www.lasercomp.com

[30] EN ISO 12667:2001. Thermal performance of building materials and products. Determination of thermal resistance by means of guarded hot plate and heat flow meter methods. Products of high and medium thermal resistance, European Committee for Standardization, 2001.

[31] T. Haavi, B. Jelle, A. Gustavsen, S. Uvsløkk, and R. Baetens, Vacuum Insulation Panels in Wood Frame Wall Constructions-Hot Box Measurements and Numerical Simulations, BEST2 Conference, Portland, Oregon, USA, 12-14 April, 2010.

[32] EN ISO 6946:2007. Building components and building elements - Thermal resistance and thermal transmittance - Calculation method, European Committee for Standardization, 2007.

[33] S. Brunner, T. Stahl, K. Ghazi Wakili, Single and double layered vacuum insulation panels of the same thickness in comparison, Best3, conference paper, 2012.

[34] M. Erb, S. Brunner, Vacuum Insulation for Building Applications. Product Declaration and Design Values, Technical report, 2012. doi: 10.13140/2.1.1171.8720.

[35] C. Sprengard, A.H. Holm, Determination of linear thermal transmittance of Vaccum Insulation Panels by measurement in a Guarded Hot-Plate (GHP) or a Heat Flow Meter (HFM) apparatus; IVIS2015 Proceedings of 12th International Vacuum Insulation Symposium, Nanjing, China, 19-21 September, 2015, pp. 292-295 


\section{FIGURE CAPTIONS}

Fig. 1. Calculation scheme for the linear thermal transmittance of the thermal bridge, $\psi$, for a portion of the envelope component having a width of $\left(I_{x}+l_{z}\right)$ and a depth of $I_{y}$. The black thick line represents the thermal bridge.

Fig. 2. (a) LASERCOMP FOX600; (b) Plates size and measurement area $A_{m}$ (dashed in red).

Fig. 3. Experimental investigation: (a) photographic survey apparatus, (b) specimens, (c) scheme of the air joint geometry.

Fig. 4. Schemes of the VIPs envelope adopted for the analysis (layers thickness in [mm]).

Fig. 5. Schemes of the VIPs assemblies adopted for the analysis: (a) Air joint; (b) Structural joint.

Fig. 6. Influence of the air joint width (d) on the value of $\psi$. Experimental ad numerical results for 10, 20, 30 $\mathrm{mm}$ thick panels.

Fig. 7. $\psi$ versus $R_{\text {joint. }}$ Experimental and numerical results.

Fig. 8. $\lambda_{\text {eq }}$ as a function of the panel size and shape $(P / A)$, with identification of the most used panel sizes (600x600 mm and 600×1200 mm) - $20 \mathrm{~mm}$ panel thickness. (Bold VIP size is the one tested. For all the other sizes "EXPERIMENTAL" values were calculated extrapolating the measured data.)

Fig. 9. Experimental and numerical linear thermal transmittance as a function of VIP thickness: (a) Politecnico di Torino results (air joints width $d \approx 5.6 \mathrm{~mm}$ ). (b) Ghazi Wakili, Bundi and Binder [3] results.

Fig. 10. Actual and ideal air gap configuration between adjacent panels. 


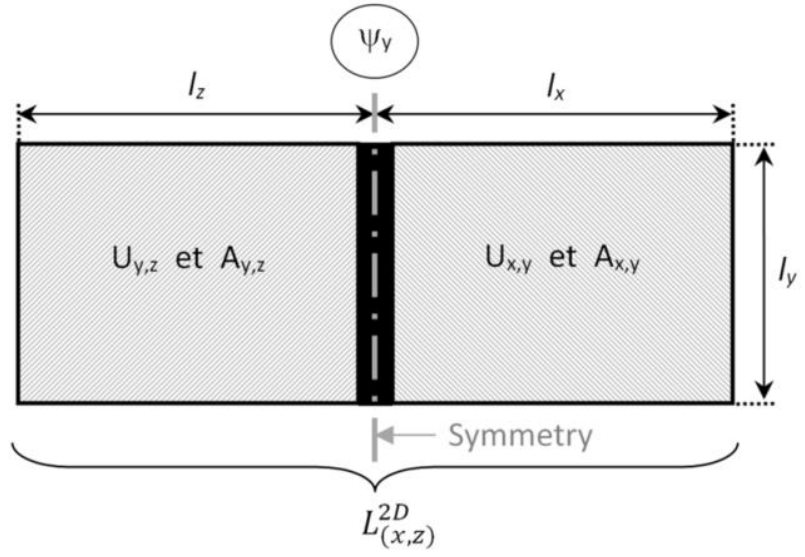

Figure 1 

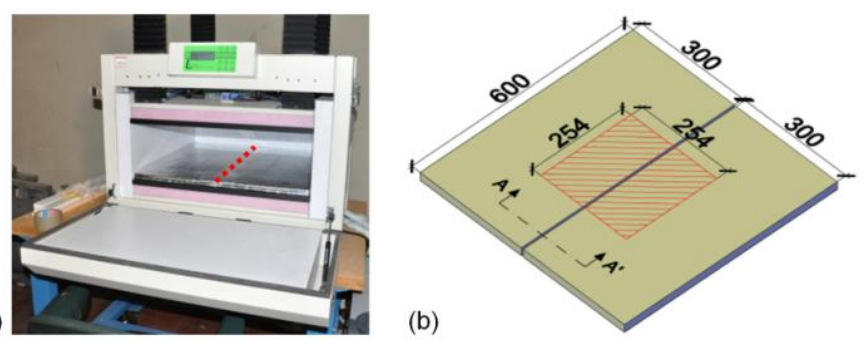

Figure 2 


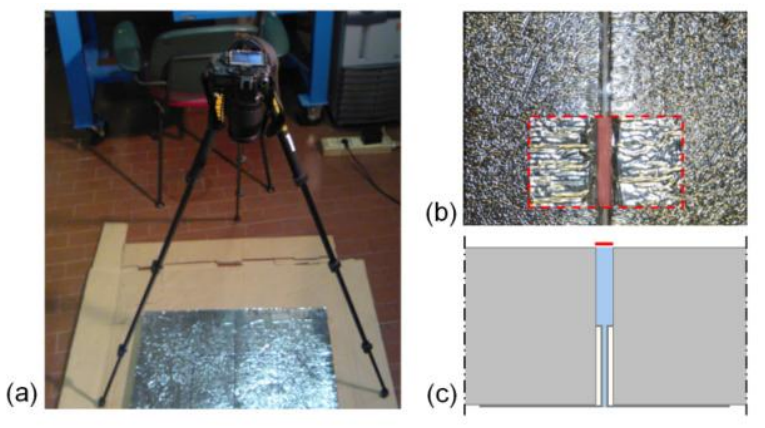

Figure 3 


\begin{tabular}{llll}
\hline & $\begin{array}{c}\text { Envelope } \\
\text { layers }\end{array}$ & $\left.\begin{array}{c}\text { s } \\
{[\mu \mathrm{m}]}\end{array}\right]$ \\
{$[\mathrm{W} / \mathrm{mK}]$}
\end{tabular}

Figure 4 


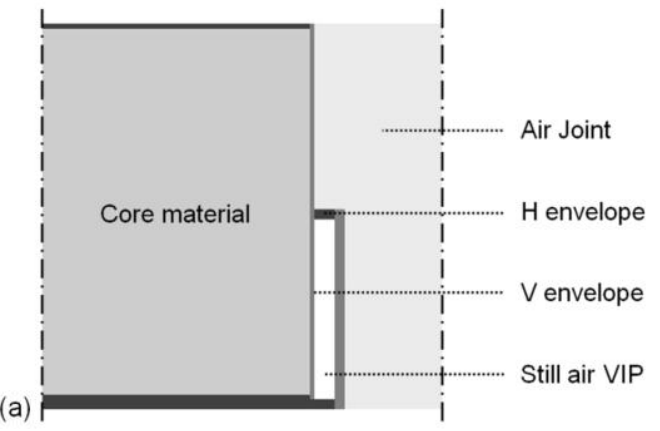

Figure 5

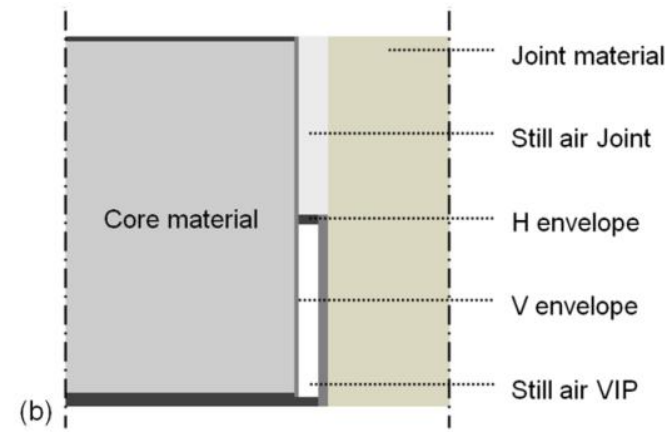

(b) 


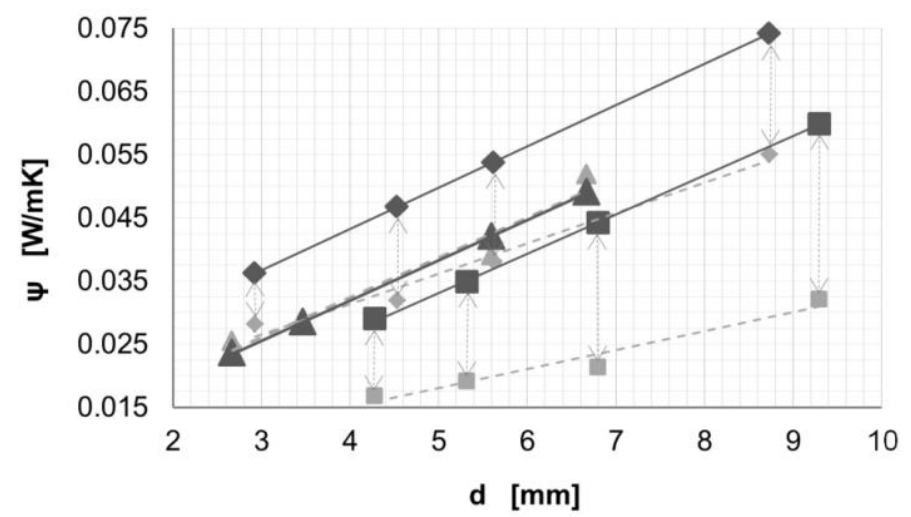

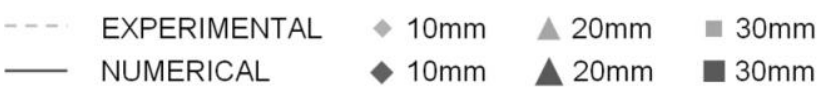

Figure 6 


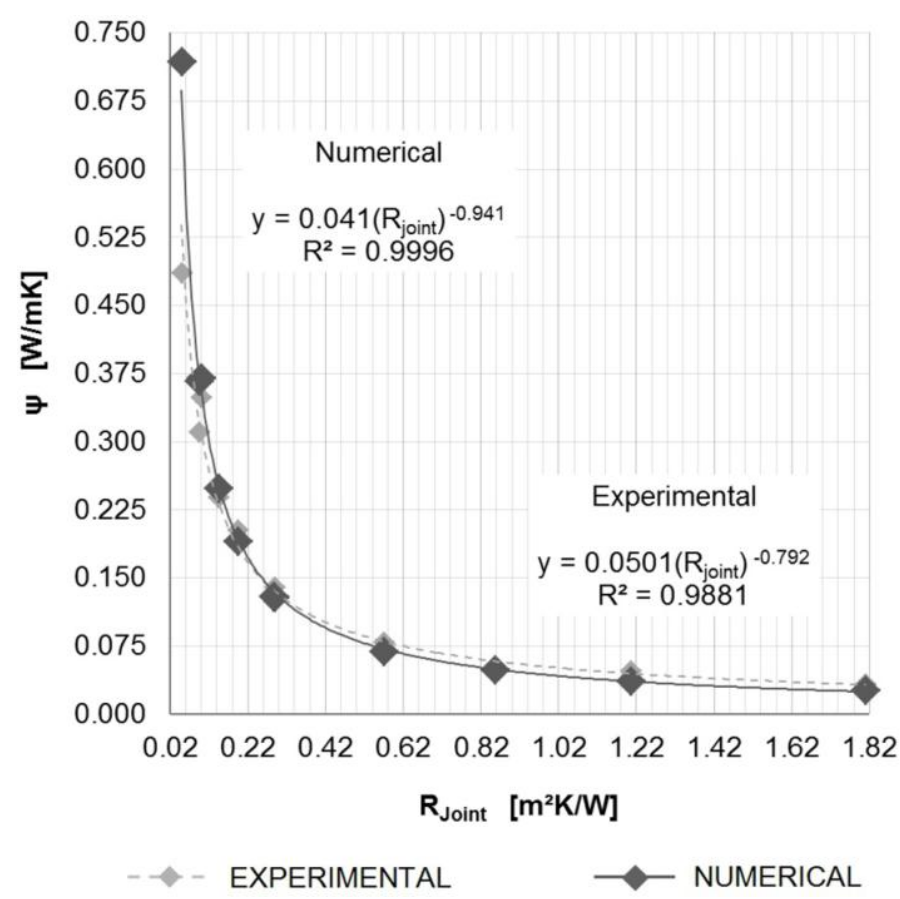

Figure 7 


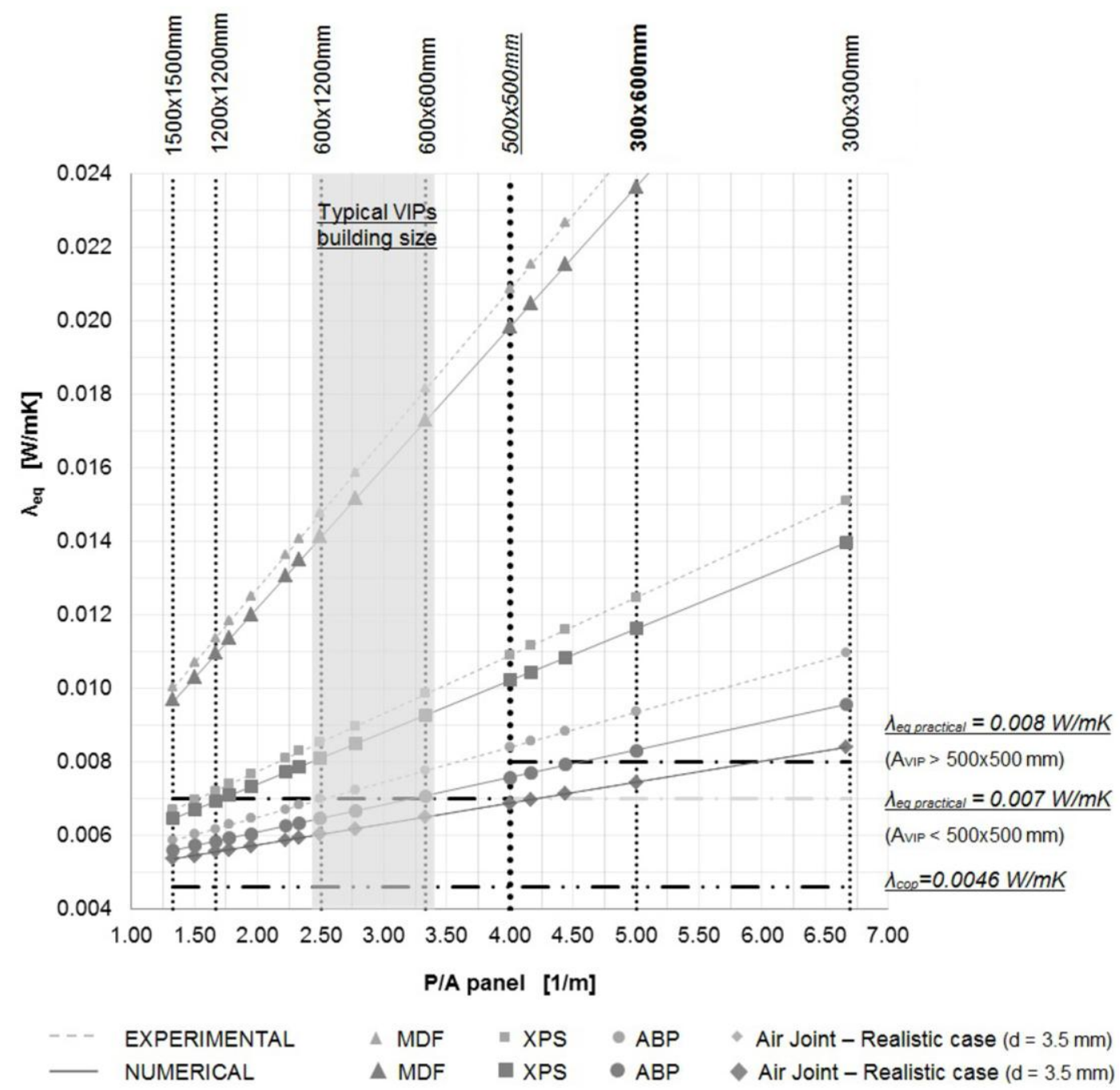

Figure 8 


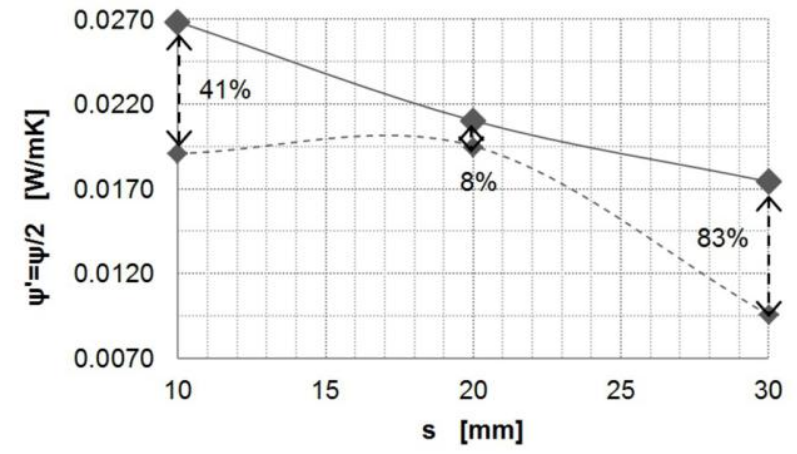

(a) $\quad$ NUMERICAL Politenico di Torino

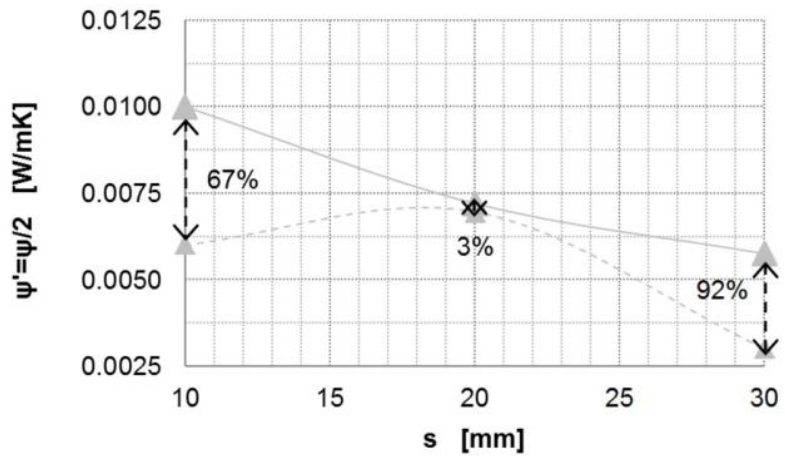

(b)
EXPERIMENTAL [3] NUMERICAL [3]

Figure 9 


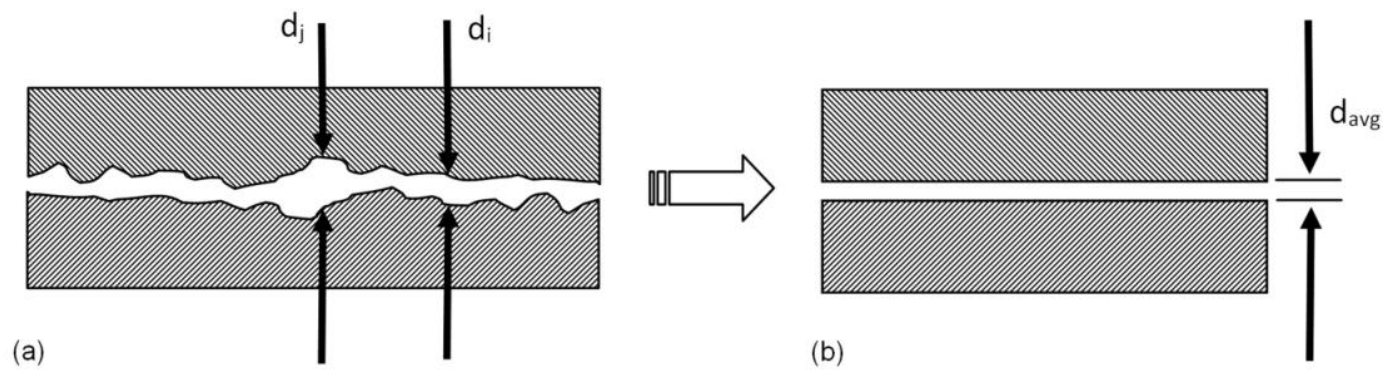

Figure 10 